Louisiana State University

LSU Digital Commons

Faculty Publications

Department of Physics \& Astronomy

6-20-2003

\title{
Bowen fluorescence from the companion star in X1822-371
}

\author{
J. Casares \\ Instituto Astrofisico de Canarias \\ D. Steeghs \\ Harvard-Smithsonian Center for Astrophysics \\ R. I. Hynes \\ The University of Texas at Austin \\ P. A. Charles \\ University of Southampton \\ K. O'Brien \\ European Southern Observatory Santiago
}

Follow this and additional works at: https://digitalcommons.Isu.edu/physics_astronomy_pubs

\section{Recommended Citation}

Casares, J., Steeghs, D., Hynes, R., Charles, P., \& O'Brien, K. (2003). Bowen fluorescence from the companion star in X1822-371. Astrophysical Journal, 590 (2 I), 1041-1048. https://doi.org/10.1086/ 375055

This Article is brought to you for free and open access by the Department of Physics \& Astronomy at LSU Digital Commons. It has been accepted for inclusion in Faculty Publications by an authorized administrator of LSU Digital Commons. For more information, please contact ir@lsu.edu. 


\title{
BOWEN FLUORESCENCE FROM THE COMPANION STAR IN X1822-371
}

\author{
J. CASARES \\ Instituto de Astrofísica de Canarias, 38200 La Laguna, Tenerife, Spain; jcv@1l.iac.es \\ D. STEEGHS ${ }^{1}$ \\ Harvard-Smithsonian Center for Astrophysics, 60 Garden Street, MS-67, Cambridge, MA 02138; \\ dsteeghs@cfa.harvard.edu \\ R. I. HYNES ${ }^{1,2}$ \\ Astronomy Department, University of Texas at Austin, 1 University Station C1400, Austin, TX 78712; \\ rih@obelix.as.utexas.edu \\ P. A. Charles \\ Department of Physics and Astronomy, University of Southampton, Southampton SO17 1BJ, UK; \\ pac@astro.soton.ac.uk \\ AND \\ K. O'BRIEN ${ }^{3}$ \\ European Southern Observatory, Casilla 19001, Santiago 19, Chile; kobrien@eso.org \\ Received 2003 January 31; accepted 2003 February 28
}

\begin{abstract}
We present clear evidence for the motion of the companion star in the low-mass X-ray binary (LMXB) X1822-371. We detect N III $\lambda 4640$ emission moving in antiphase with the radial velocity curve of the neutron star and produced on the X-ray-heated hemisphere of the donor star. From the motion of this feature we derive a lower limit to the radial velocity semiamplitude of the companion star, $K_{2} \geq 300 \pm 8 \mathrm{~km} \mathrm{~s}^{-1}$, which, combined with a previous determination of the inclination angle and the pulsar's radial velocity curve, yields $M_{2} \geq 0.36(2) M_{\odot}$ and $M_{1} \geq 1.14(6) M_{\odot}$. The He I $\lambda 4471$ absorption line moves at lower velocities $(\simeq 225$ $\mathrm{km} \mathrm{s}^{-1}$ ) and with a -0.05 phase shift, suggesting a likely origin on the gas stream near the L1 point. In addition, we detect an S-wave emission of $\mathrm{O}$ VI $\lambda 3811$ produced by illumination of the hot spot bulge by the central source. The Balmer lines are dominated by broad absorptions probably due to obscuration of the accretion disk by vertically extended cool material from the splash region and overflowing stream. We also derive a more accurate, and significantly different (compared to earlier work), systemic velocity of $\gamma=-44 \pm 5 \mathrm{~km} \mathrm{~s}^{-1}$, based on the motion of the He II $\lambda 4686$ wings and Doppler tomography. This work confirms the power of imaging the companion stars in LMXBs and outbursting transients using the Bowen fluorescence transitions.
\end{abstract}

Subject headings: accretion, accretion disks — binaries: close — stars: individual (X1822-371) — X-rays: binaries $-\mathrm{X}$-rays: stars

On-line material: color figures

\section{INTRODUCTION}

$\mathrm{X} 1822-371$ is one of the brightest low-mass X-ray binaries (LMXBs) in the optical, but it is comparatively weak in X-rays (i.e., $L_{\mathrm{X}} / L_{\mathrm{opt}}=20$; it is usually $\sim 500-1000$; see van Paradijs \& McClintock 1995), which makes it the prototypical accretion disk corona (ADC) source. In ADC sources, the combination of a high inclination and a thick disk obscures the central source, and only X-rays scattered from material above and below the disk (a "corona") can reach the observer (White \& Holt 1982). The optical light curve displays regular eclipses of the accretion disk by the companion star on a $5.57 \mathrm{hr}$ orbital period (see, e.g., Hellier \& Mason 1989). Detailed modeling of the optical and X-ray light curves has provided an accurate determination of the inclination angle, $i=82^{\circ} .5 \pm 1.5$ (Heinz \& Nowak 2001; see also Hellier \& Mason 1989).

\footnotetext{
${ }^{1}$ Department of Physics and Astronomy, University of Southampton, Southampton SO17 1BJ, UK.

${ }^{2}$ Hubble Fellow.

${ }^{3}$ School of Physics and Astronomy, University of St. Andrews, St. Andrews KY16 9SS, UK.
}

$\mathrm{X} 1822-371$ is also remarkable thanks to the discovery of 0.59 s X-ray pulses (Jonker \& van der Klis 2001, hereafter $\mathrm{JvdK}$ ), which flags it as one of the "rare" precursors of millisecond pulsars (eight out of several hundred LMXBs). The analysis of the pulse arrival time delay has enabled an extremely precise determination of the orbit of the neutron star: $a \sin i=1.006 \mathrm{lt}-\mathrm{s}$, corresponding to a primary radial velocity semiamplitude of $94.5 \pm 0.5 \mathrm{~km} \mathrm{~s}^{-1}$.

A complete determination of the system parameters, however, requires the knowledge of the radial velocity curve of the companion star. This was first intimated by Harlaftis, Charles, \& Horne (1997) from the motion of He I $\lambda 5876$ absorption, which was interpreted as being produced on the irradiated face of the companion star, thereby providing a lower limit to the velocity semiamplitude of $K_{2} \geq 225 \pm 23 \mathrm{~km} \mathrm{~s}^{-1}$. More recently, Jonker, van der Klis, \& Groot (2003) report $K_{2}=327 \pm 17$ $\mathrm{km} \mathrm{s} \mathrm{s}^{-1}$, based on measurements of $\mathrm{He}_{\mathrm{I}} \lambda \lambda 4026$ and 5875. However, they also find a -0.08 phase shift with respect to the pulse-timing ephemeris, which they attribute to asymmetric irradiation of the companion's Roche lobe, and a systemic velocity of $67 \pm 15 \mathrm{~km} \mathrm{~s}^{-1}$, 
TABLE 1

LOG OF THE OBSERVATIONS

\begin{tabular}{ccccc}
\hline \hline & & $\begin{array}{c}\text { Exposure } \\
\text { Time } \\
\text { Date (2002) }\end{array}$ & $\begin{array}{c}\text { Wavelength } \\
\text { Range } \\
(\AA \text { Tixel }\end{array}$ & \\
\hline Jun $10 \ldots \ldots \ldots \ldots . . \ldots$ & AAT & $24 \times 900$ & $3650-5100$ & Dispersion \\
Jun $11 \ldots \ldots \ldots \ldots . .$. & AAT & $14 \times 900$ & $3650-5100$ & 0.45 \\
& NTT & $1 \times 1200$ & $4400-5150$ & 0.45 \\
& & $4 \times 600$ & $4400-5150$ & 0.40 \\
\hline
\end{tabular}

significantly larger than that of Harlaftis et al. $(19 \pm 19$ $\mathrm{km} \mathrm{s}^{-1}$.

In this paper, we present new phase-resolved, intermediate-resolution blue spectroscopy of X1822-371. Inspired by our work on the LMXB Sco X-1 (Steeghs \& Casares 2002, hereafter SC), we are pursuing the detection of the companion star via Bowen fluorescence emission due to $\mathrm{X}$-ray irradiation. The combination of velocity information and light curves enable us to locate the site of the main emission/absorption lines within the binary.

\section{OBSERVATIONS AND DATA REDUCTION}

We observed X1822-371 using the RGO spectrograph attached to the $3.9 \mathrm{~m}$ Anglo-Australian Telescope (AAT) on the nights of 2002 June 10-11. A total of 38 spectra were obtained with the R1200B grating centered at $4350 \mathrm{~A}$, which provided a wavelength coverage of 3500-5250 $\AA$, although the extreme $150 \AA$ at each end were severely affected by vignetting and not employed in the analysis. The seeing was variable $\left(1 " .1-2^{\prime \prime}\right)$ during our run, and we used a $1^{\prime \prime}$ slit, which resulted in a resolution of $70 \mathrm{~km} \mathrm{~s}^{-1}$ (FWHM). The flux standard Feige 15 was observed with the same instrumental configuration, so as to correct for the instrumental response of the detector. We also rotated the slit to include a comparison star, which enabled us to monitor slit losses and obtain a relative flux calibration. In addition, we obtained five more spectra of X1822-371 with EMMI and grating No. 6 on ESO'S 3.5 m New Technology Telescope (NTT) at La Silla on 2002 June 11. These spectra cover the spectral range $4400-5150 \AA$ at $75 \mathrm{~km} \mathrm{~s}^{-1}$ resolution. In total we covered 1.5 orbital cycles of the target on June 10 and one cycle on June 11. Table 1 contains a log of the observations.

The images were debiased and flat-fielded and the spectra subsequently extracted using conventional optimal extraction techniques in order to optimize the signal-to-noise ratio of the output (Horne 1986). CuAr comparison lamp images were obtained every 30 minutes, and the $\lambda$-pixel scale was derived through fifth-order polynomial fits to 95 lines, resulting in an rms scatter of less than $0.025 \AA$. The calibration curves were interpolated linearly in time.

\section{TRAILED SPECTRA AND RADIAL VELOCITY CURVES}

Absolute flux calibration of X1822-371 and its slit comparison was tied to the spectrophotometric standard Feige 15. Slit losses for individual spectra were computed by fitting a second-order spline to the ratio between each comparison star spectrum and its grand sum average. The X1822-371 spectra were subsequently divided by their corresponding splines in order to get the final fluxed spectra,

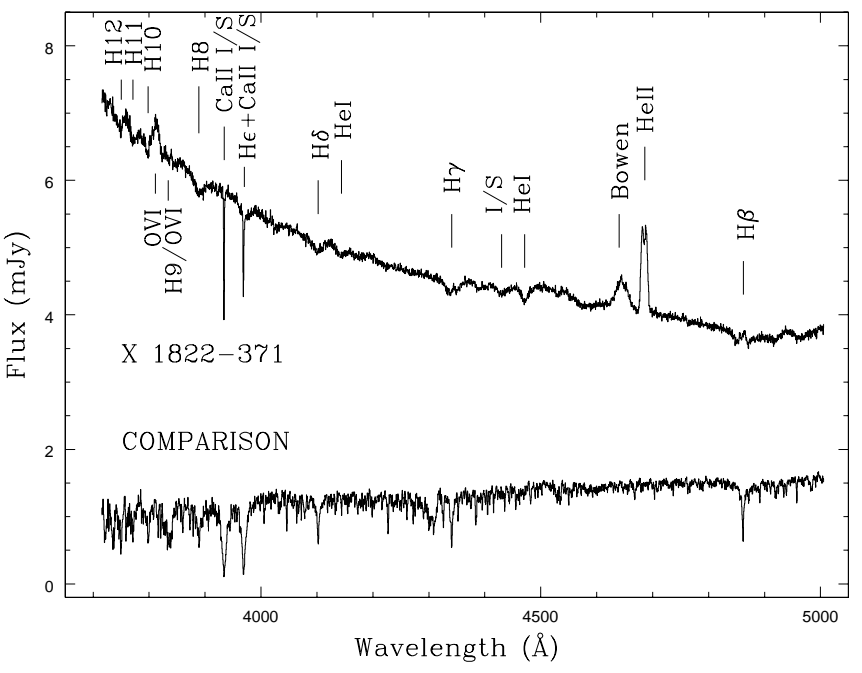

FIG. 1.-Average optical spectrum of X1822-371 and the comparison star in the slit. The main emission and absorption features are indicated.

corrected for slit losses. Figure 1 shows the average spectra of X1822-371 and its slit comparison in millijansky flux units. The spectrum is dominated by high-excitation emission lines of He II $\lambda 4686$ and the Bowen blend. There is also evidence of a broad emission feature at $\simeq 3813 \AA$, which was already noted by Charles, Thorstensen, \& Barr (1980) in their low-resolution spectra and tentatively identified with either He II $\lambda 3813$ or O vi $\lambda 3811$ (see, however, Mason et al. 1982 for a different interpretation). The He I and Balmer lines are dominated by broad absorptions, with residual emission cores observable in $\mathrm{H} \beta$ and $\mathrm{H} \gamma$.

Orbital phases were computed using the most accurate quadratic ephemeris, published by Parmar et al. (2000). However, given the large number of cycles we estimate an accumulated uncertainty of 0.03 orbits at the time of our observations, and hence we decided to take the most updated $T_{0}$ from JvdK. This is defined by the superior conjunction of the X-ray pulsar and yields a final accuracy of 0.002 cycles. Binary phases are therefore computed from the ephemeris

$$
\begin{aligned}
T= & 2,450,993.27968+0.232108785(50) E \\
& +2.06(23) \times 10^{-11} E^{2} .
\end{aligned}
$$

The orbital evolution of several lines is plotted in Figure 2 in the form of trailed spectrograms. Balmer lines are dominated by deep absorptions, with velocity shifts of $\sim 800 \mathrm{~km}$ $\mathrm{s}^{-1}$, maximum depth between phases 0.7 and 1.0 , and strengthening with excitation energy. Some residual emission cores are nonetheless seen in $\mathrm{H} \beta$ and $\mathrm{H} \gamma$ at phase $0-0.1$. The He I lines are always seen in absorption with lower amplitudes $\left(\sim 200 \mathrm{~km} \mathrm{~s}^{-1}\right)$ and blue-to-red crossing velocities at phase 0 (e.g., see $\mathrm{He}$ I $\lambda 4471)$. A radial velocity curve is presented in the top panel of Figure 3, obtained after cross-correlating the spectra, co-added in 13 phase bins, with a Gaussian of FWHM $=500 \mathrm{~km} \mathrm{~s}^{-1}$. As noted by Harlaftis et al. (1997; see also Jonker et al. 2003), the radial velocity curve is distorted around phase 0 because of possible contamination by another component. Therefore, we mask the data points within the phase interval 0.95 0.25 and perform a sine wave fit to the remaining data to derive the following parameters: $\gamma=24 \pm 28 \mathrm{~km} \mathrm{~s}^{-1}$, 

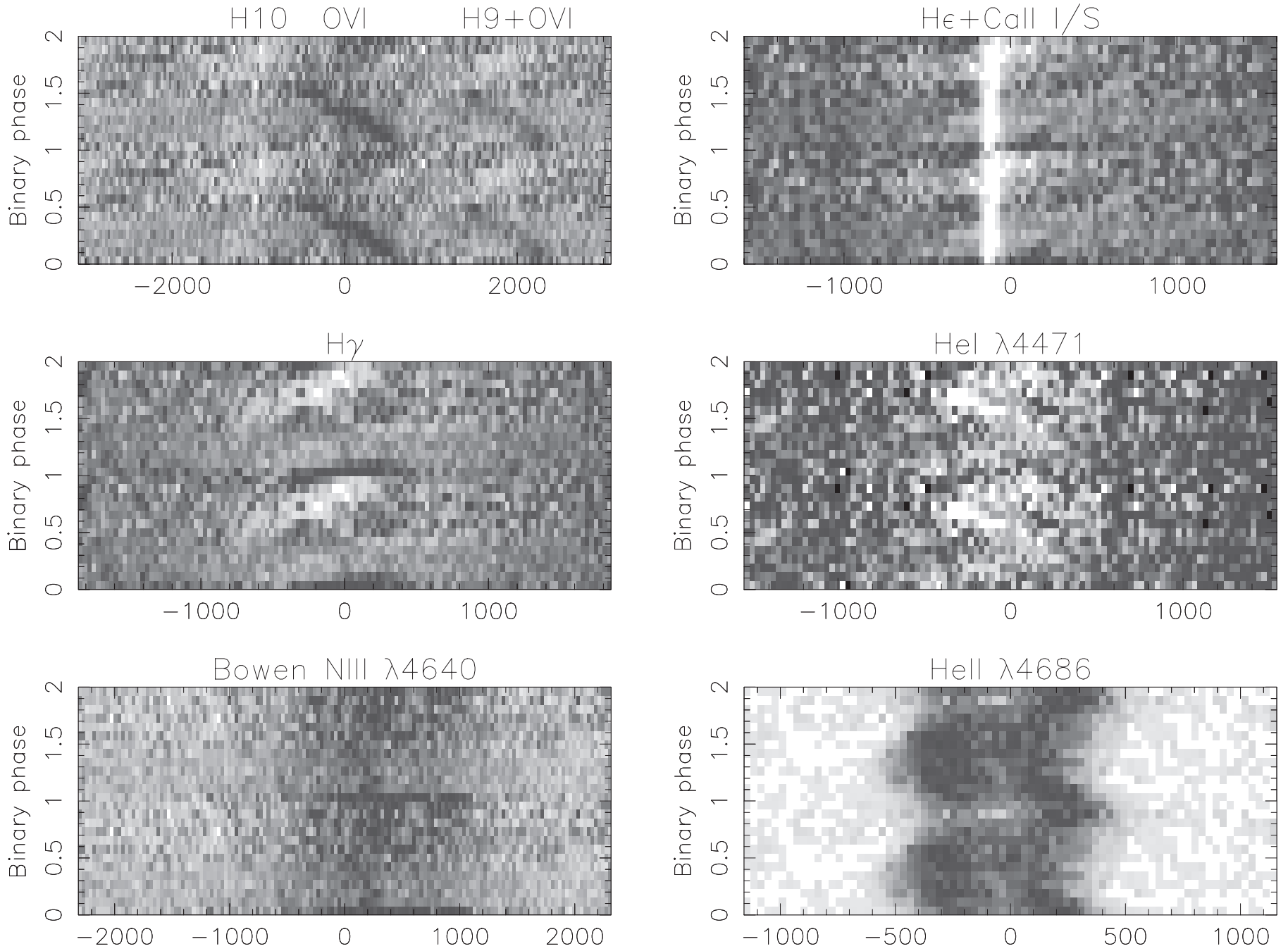

FIG. 2.-Trailed spectrogram of selected lines folded in 13 phase bins. [See the electronic edition of the Journal for a color version of this figure.]

$V=229 \pm 37 \mathrm{~km} \mathrm{~s}^{-1}$, and $\Phi_{0}=-0.07 \pm 0.02$, where $V$ is the velocity semiamplitude and $\Phi_{0}$ the blue-to-red crossing. These numbers are in good agreement with Harlaftis et al. (1997), and the phase shift confirms the claim by Jonker et al. (2003) that the radial velocity curve of He I occurs earlier than expected from the pulse-timing ephemeris.

The He II $\lambda 4686$ emission exhibits a classic double-peaked disk profile, with an S-wave crossing from blue to red velocities at phase $\sim 0.8$. We postpone a detailed analysis of the radial velocity curve of $\mathrm{He}$ II to $\S 5$. On the other hand, the $\lambda 3813$ line displays a single S-wave, significantly broader $\left(\mathrm{FWHM} \sim 450 \mathrm{~km} \mathrm{~s}^{-1}\right.$ ) than our resolution, and blue-tored crossing also at phase 0.8 . The S-wave pattern of this feature in our trailed spectra demonstrates that it is a real line and not just a spurious feature caused by the broadened Balmer absorptions at each side (Mason et al. 1982). As noted by Charles et al. (1980), if it were He II $\lambda 3813$ there would be no special reason why this line should be enhanced relative to others in the Pickering series. Alternatively, it could be the blue member of the O vi doublet $\lambda \lambda 3811,3834$, and indeed we see another S-wave emission in our trailed spectra at $\sim 3834 \AA$, although much weaker because of overlapping with the broad H9 absorption. Therefore, we identify this feature with $\mathrm{O}$ vi $\lambda 3811.4$. We have also extracted the radial velocity curve of this line by cross-correlation of our 13 phase bins of co-added spectra with a Gaussian of $\mathrm{FWHM}=600 \mathrm{~km} \mathrm{~s}^{-1}$, and the results are presented in Figure 3 (middle panel). A sine wave fit yields $\gamma=46 \pm 24$ $\mathrm{km} \mathrm{s}^{-1}, V=370 \pm 30 \mathrm{~km} \mathrm{~s}^{-1}$, and $\Phi_{0}=0.82 \pm 0.01$. The phasing and large velocity amplitude suggest an origin related to the accretion disk/hot spot region.

The Bowen blend shows a complicated pattern of narrow (FWHM $\sim 100 \mathrm{~km} \mathrm{~s}^{-1}$ ) S-waves, barely detectable in our limited signal-to-noise ratio individual spectra, superposed on the broad disk emission. These narrow components are identified with the Bowen fluorescent components of $\mathrm{N}$ III $\lambda \lambda 4634.12,4640.64$ and $C_{\text {III }} \lambda \lambda 4647.4,4650.1$ and have been shown to arise from the irradiated hemisphere of the donor in Sco X-1 (see SC). We have attempted to extract radial velocities of these sharp Bowen fluorescent components using a multi-Gaussian fit, as explained in SC, but the method failed because of the weakness of these features in the individual spectra. However, in $\S 6$ we show how we can exploit the powerful Doppler tomography technique (Marsh 2001) to derive the radial velocity curve of the $\mathrm{N}$ III $\lambda 4640.64$ line, which is found to trace the motion of the donor star. The reliability of this technique was already demonstrated in the case of Sco X-1; radial velocities 


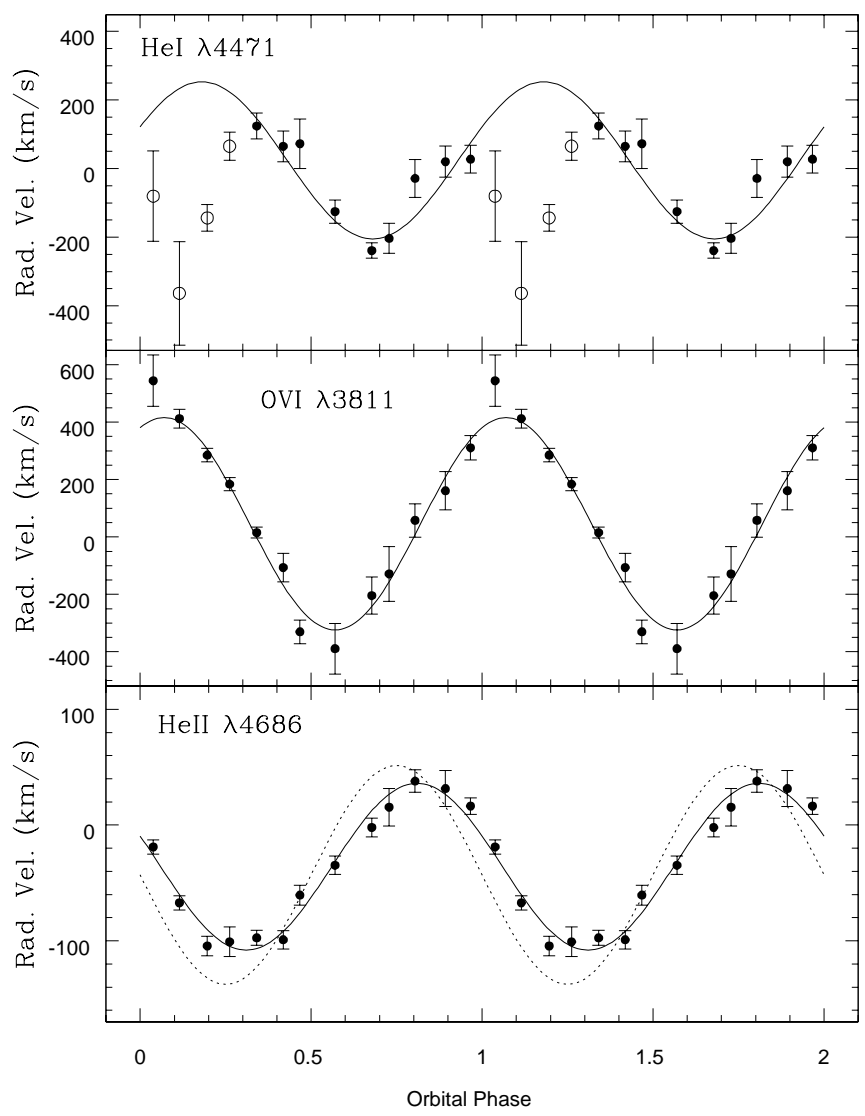

Fig. 3.-Radial velocity curves of He I $\lambda 4471$ (top), O vi $\lambda 3811$ (middle), and the wings of He II $\lambda 4686$ (bottom). The latter was obtained by crosscorrelation with a double-Gaussian template of FWHM $=100 \mathrm{~km} \mathrm{~s}^{-1}$ and separation $a=900 \mathrm{~km} \mathrm{~s}^{-1}$ (see $\S 5$ for details). Best sine wave fits are shown, after masking a few outliers, marked with open circles, for He I $\lambda 4471$ (see text for details). The dotted line displays the radial velocity curve of the neutron star, using the pulse-timing ephemeris from JvdK.

derived from Doppler maps were in excellent agreement with the results from Gaussian fitting (SC).

\section{LIGHT CURVES}

In the top left-hand panel of Figure 4, we show the continuum light curve, extracted from integrating the individual fluxed spectra in the wavelength range 4000-4600 A. Filled circles correspond to the night of July 10 and open circles to that of July 11. In addition to the eclipse of the disk by the companion star, we also note a posteclipse hump centered at orbital phase $0.25-0.3$, which has been interpreted as the visibility of the irradiated inner disk rim or hot spot hump (see, e.g., Mason \& Córdova 1982). We note that this is when the $\mathrm{O}$ vi flux (top right-hand panel) is also at a maximum, suggesting that it too is located in this region.

We have also obtained integrated light curves of the main emission/absorption lines, and these are also presented in Figure 4 . The light curve of He II $\lambda 4686$ shows a narrow eclipse at phase 0.2 and maximum emission around phase 0.75 , suggesting that the bulk of the emission might be related to the visibility of the stream/disk impact region (i.e., equivalent to the classical hot spot in a cataclysmic variable). The Bowen blend exhibits a sine wave modulation peaking at phase 0.5 , which suggests a likely origin on the irradiated hemisphere of the donor star. We note that the phases of maximum emission of both $\mathrm{He}$ II and Bowen are the same as observed in the neutron star transient XTE J2123-058 during its outburst phase (Hynes et al. 2001).

The $\mathrm{O}$ vi $\lambda 3811$ emission line shows a light curve similar to that of the Bowen blend, but leading in phase by $\sim 0.15$ orbits. The minimum flux occurs at phase 0.8 , which also coincides with the blue-to-red crossing (see Fig. 3). The combination of the light curve and velocity information strongly suggests that $\mathrm{O}$ vi $\lambda 3811$ is associated with the irradiated inner rim of the hot spot bulge, which is self-obscured by the outer disk rim at phase $\simeq 0.8$. The amplitude of the modulation is a factor of 2 larger than in the Bowen blend, and also the flux drops to zero at minimum, indicating that the entire line is produced at the irradiated inner bulge, with no significant contribution from other regions (e.g., the axisymmetric disk or a companion star). None of the emission lines exhibit a sharp eclipse feature at phase 0 , and hence they must arise in a vertically extended structure, such as a disk atmosphere or corona.

The Balmer lines show intricate light curves in absorption. They exhibit two minima, at phases 0.25 and 0.8 , increasing in depth with excitation energy, i.e., deeper for $\mathrm{H} 8$ than for $\mathrm{H} \beta$. The trailed spectra of $\mathrm{H} \gamma$ in Figure 2 clearly show that the phase 0.25 dip is produced by a sharp absorption, whereas the 0.8 minimum is caused by a broader feature moving redward from phase 0.5 to 1.0 . The phase 0.8 absorption is consistent with obscuration of the irradiated inner disk by a high disk rim extending from phase 0.5 to 0.9 . The light curve of $\mathrm{He}$ I $\lambda 4471$, also in absorption, appears to modulate only weakly with orbital phase, reaching maximum strength around phase 0.6 .

\section{THE SYSTEMIC VELOCITY}

Before discussing the location of the different emission sites, we need to estimate the systemic velocity from these data. So far there is no clear determination, with only tentative values based on radial velocity curves of the $\mathrm{He}$ I lines: $19 \pm 19 \mathrm{~km} \mathrm{~s}^{-1}$ (Harlaftis et al. 1997) and $67 \pm 15 \mathrm{~km} \mathrm{~s}^{-1}$ (Jonker et al. 2003). On the other hand, we notice that the strong He II $\lambda 4686$ emission is blueshifted in our sum spectrum, and a simple Gaussian fit yields a centroid position at $-50 \mathrm{~km} \mathrm{~s}^{-1}$. The double-peaked profile indicates that it comes from the accretion disk, and hence it will likely provide the most reliable $\gamma$ determination.

In an attempt to estimate both the systemic velocity and the velocity semiamplitude of the compact object $K_{1}$, we have applied the double-Gaussian technique to $\mathrm{He}$ II $\lambda 4686$ (Schneider \& Young 1980). We employed a twoGaussian bandpass with FWHM $=100 \mathrm{~km} \mathrm{~s}^{-1}$ and Gaussian separations $a=400-1300 \mathrm{~km} \mathrm{~s}^{-1}$ in steps of $100 \mathrm{~km} \mathrm{~s}^{-1}$. The $K_{1}$ velocity drops dramatically from 230 to $\simeq 60 \mathrm{~km} \mathrm{~s}^{-1}$ as we move from the line core to the wings, whereas the systemic velocity yields consistent values in the range -30 to $-60 \mathrm{~km} \mathrm{~s}^{-1}$. We find that the velocity points start to be corrupted by continuum noise for $a>900 \mathrm{~km} \mathrm{~s}^{-1}$, and a sine wave fit (with the period fixed) to this velocity curve gives $K_{1}=63 \pm 3 \mathrm{~km} \mathrm{~s}^{-1}$, $\phi_{0}=0.58 \pm 0.01$, and $\gamma=-43 \pm 4 \mathrm{~km} \mathrm{~s}^{-1}$ (see bottom panel of Fig. 3). On the other hand, the radial velocity curve of the compact object is well determined through the pulse-timing analysis and yields $K_{1}=94.5 \pm 0.5 \mathrm{~km}$ $\mathrm{s}^{-1}$ (represented by a dotted curve in Fig. 3). The small difference in phasing $(0.08$ orbits $)$ and $K_{1}$ velocity 

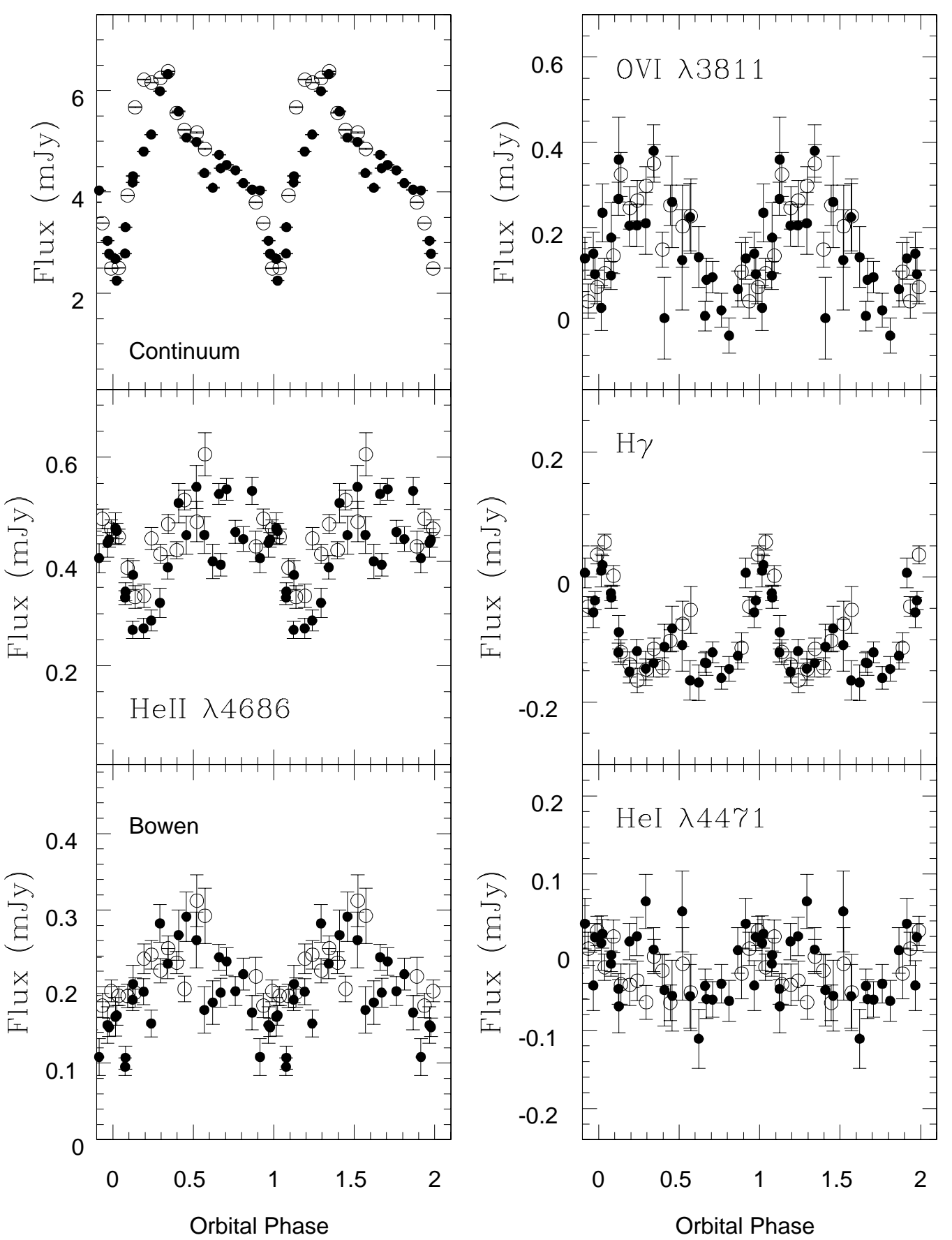

FIG. 4. - Light curves of the continuum (4000-4600 $\AA$ ) and selected emission/absorption lines. Filled and open circles correspond to the nights of June 10 and 11 , respectively.

indicates that even the line wings are contaminated by inhomogeneities in the disk. Nevertheless, the systemic velocity appears quite stable, and hereafter in this paper we adopt $\gamma=-43 \mathrm{~km} \mathrm{~s}^{-1}$ from the fit using $a=900 \mathrm{~km}$ $\mathrm{s}^{-1}$. This is subject to systematic uncertainties, but further confirmation for a systemic velocity close to -43 is presented in the next section. At this point, we note that the radial velocity curve of $\mathrm{O}$ VI implies a positive systemic velocity of $\sim 43 \mathrm{~km} \mathrm{~s}^{-1}$ (see $\S 3$ ). This is due to its formation in gas located on the irradiated inner face of the hot spot, which probably has a local vertical velocity component after the gas stream collision with the disk outer edge (see Fig. 5).

\section{DOPPLER MAPPING}

Armed with this value of $\gamma$, we have computed Doppler maps of the principal lines seen in our spectra, and these are presented in Figure 5. All the spectra were rectified, by subtracting a low-order spline fit to the continuum, and rebinned into a uniform velocity scale of $15 \mathrm{~km} \mathrm{~s}^{-1}$. Doppler tomography combines the orbitally resolved line profiles to reconstruct the brightness distribution of the system in velocity space, by maximizing the entropy of the final image (see Marsh 2001 for details). Since Doppler tomography only deals with emission features, in order to map the absorption line $\mathrm{He}$ I $\lambda 4471$ we first had to invert the spectra. 

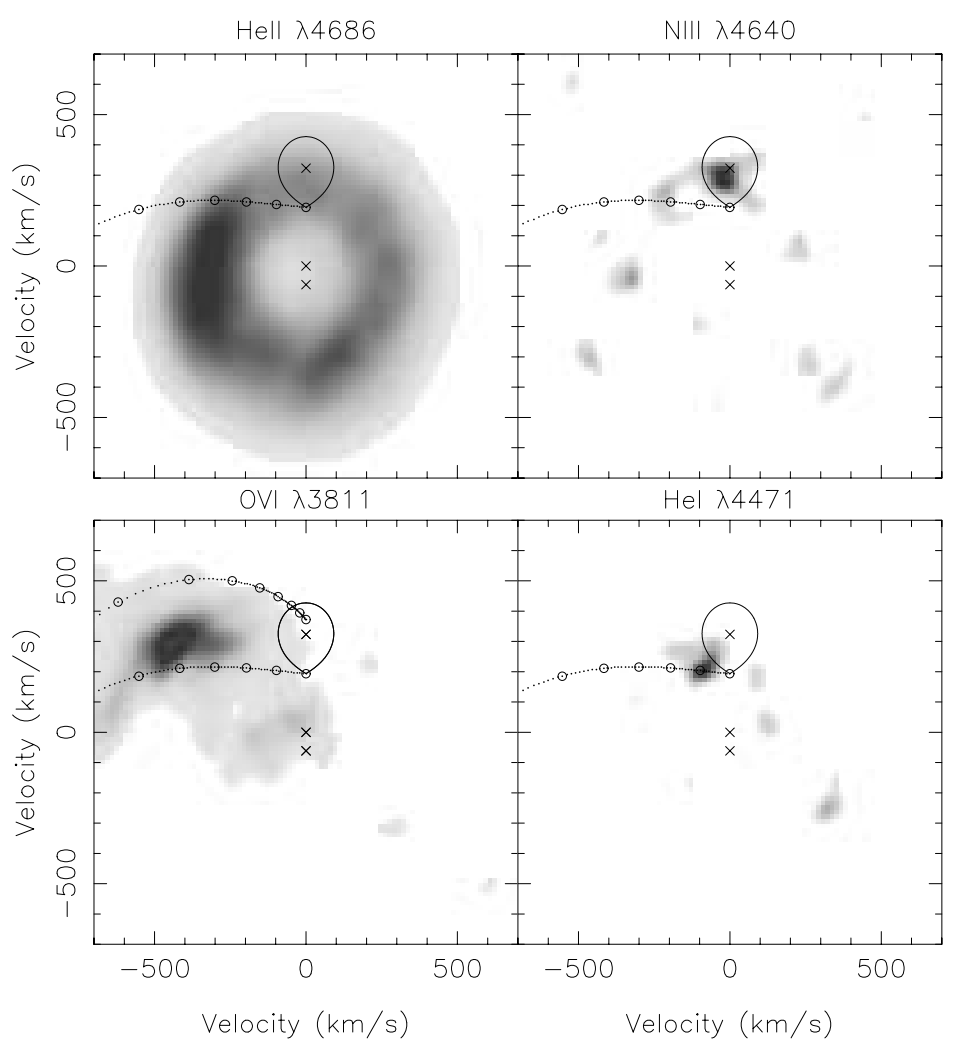

FIG. 5.-Doppler maps of several spectral features. We have overplotted the Roche lobe of the companion star, the gas stream trajectory, and the compact object's position for $q=0.29$, which assumes $M_{1}=1.4 M_{\odot}$ (i.e., $K_{2}=324 \mathrm{~km} \mathrm{~s}^{-1}$ ). In the map of O vI we also plot the Keplerian velocity of the disk along the gas stream path. All the images were computed for a systemic velocity $\gamma=-43 \mathrm{~km} \mathrm{~s}^{-1}$. [See the electronic edition of the Journal for a color version of this figure.]

The emission-line distribution of He II $\lambda 4686$ shows the classic ringlike distribution of an accretion disk. The distribution is slightly asymmetric, with maximum emissivity over an extended arc peaking at phase $\sim 0.8$. In order to help interpret the maps we have overplotted the Roche lobe of the companion star and the gas stream trajectory for a typical neutron star mass of $M_{1}=1.4 M_{\odot}$, and hence $q=M_{2} / M_{1}=K_{1} / K_{2}=0.29$. We have also marked the center of mass and the compact object's position with crosses.

We used the He II Doppler map to verify the adopted systemic velocity. The $\chi^{2}$ value of the map was calculated for a range of $\gamma$, and the best fit in terms of minimal $\chi^{2}$ was achieved for $\gamma=-44 \pm 5 \mathrm{~km} \mathrm{~s}^{-1}$. We also searched for the optimal center of symmetry of the He II map as a means to estimate $K_{1}$ (see $\mathrm{SC}$ ). We found that the disk emission was subtracted optimally by assuming a $K_{1}$ of $97 \pm 5 \mathrm{~km} \mathrm{~s}^{-1}$. We did not include the region at negative $V_{x}$ velocities, since this part of the map is severely affected by the bright spot asymmetries. This value compares favorably with the radial velocity amplitude of the primary as determined from the $\mathrm{X}$-ray pulsations ( JvdK).

The Doppler image of O VI $\lambda 3811$ shows no evidence for the accretion disk but a very compact emission knot that lies between the predicted gas stream velocity and the Keplerian velocity of the disk along the gas stream path. The $\mathrm{O}$ VI emission probably arises from irradiated turbulent gas around the hot spot, which, after the shock, shares the velocity of the gas stream and the accretion disk. This is identical to the Doppler maps of He II $\lambda 4686$ and He I $\lambda 4471$ computed for the cataclysmic variable U Gem (Marsh et al. 1990).
The Doppler analysis of the Bowen blend is complicated by the merging of many $\mathrm{C}$ III, $\mathrm{N}$ III, and $\mathrm{O}$ II transitions (see, e.g., McClintock, Canizares, \& Tarter 1975). The most prominent component is $\mathrm{N}$ III $\lambda 4640.64$, for which we have also computed a tomogram. We clearly detect a sharp spot along the $V_{y}$-axis, and we have inferred its centroid position at $V_{x} \sim-12 \pm 8 \mathrm{~km} \mathrm{~s}^{-1}$ and $V_{y} \sim 300 \pm 8 \mathrm{~km} \mathrm{~s}^{-1}$ through a two-dimensional Gaussian fit. The spot is effectively on the $V_{y}$-axis, given our phase uncertainties. The phasing and velocity amplitude proves that this emission is produced on the irradiated front side of the donor. We have experimented with computing maps for a set of $\gamma$-velocities in the range 0 to $-80 \mathrm{~km} \mathrm{~s}^{-1}$, and we find that only $\gamma$-values in the range -40 to $-60 \mathrm{~km} \mathrm{~s}^{-1}$ provide well-focused $\mathrm{N}$ III spots. The remaining $\gamma$-velocity maps produce more extended and blurred spots, which further confirm our systemic velocity determination. In Figure 6, we compare the Bowen blend average of X1822-371 and Sco X-1 (from SC) in the rest frame of the donor stars. The prominent N III $\lambda 4640$ emission and the $\mathrm{C}$ III blend at $\lambda \lambda 4647,4650$ are clearly identified in X1822-371, whereas the $\mathrm{N}$ III $\lambda 4634$ is diluted in the much noisier spectrum of X1822-371.

Finally, we also present the tomogram of $\mathrm{He}$ I $\lambda 4471$ absorption, which very much resembles the map of $\mathrm{N}$ III $\lambda 4640.64$, but the spot seems to be skewed to the leading side of the donor or on the gas stream, at $0.1 R_{\mathrm{L} 1}$ from the L1 point. A two-dimensional Gaussian fit to the bright spot yields a centroid position at $V_{x}=-74 \pm 8 \mathrm{~km} \mathrm{~s}^{-1}$ and $V_{y} \sim 224 \pm 8 \mathrm{~km} \mathrm{~s}^{-1}$, which places it exactly over the gas stream trajectory. 


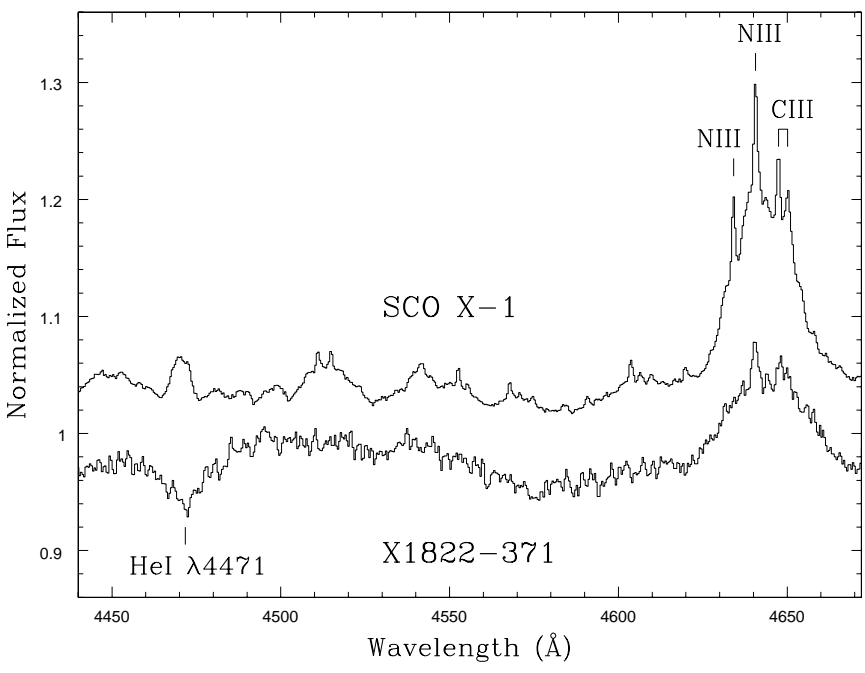

Fig. 6.-Doppler-corrected averaged spectra of Sco X-1 and $\mathrm{X} 1822-371$, in the rest frame of the companion. In the latter case we have used $\gamma=-43 \mathrm{~km} \mathrm{~s}^{-1}$ and $K_{2}=300 \mathrm{~km} \mathrm{~s}^{-1}$.

\section{DISCUSSION}

We have successfully detected the companion star in X1822-371 through Doppler imaging of the fluorescent $\mathrm{N}$ III $\lambda 4640$ emission. The emission is exactly in antiphase with the radial velocity curve of the pulsar, and hence no asymmetric irradiation of the companion's Roche lobe needs to be invoked. On the other hand, the distribution of He I $\lambda 4471$ absorption is concentrated near the L1 point but leading in phase. We find a phase shift of $\simeq-0.05$ with respect to the position of the N III $\lambda 4640$ spot and the pulsetiming ephemeris, which places the He I absorber on the leading side of the companion's Roche lobe or, more likely, over the gas stream. This solves the problem raised by Jonker et al. (2003), who, following Harlaftis et al. (1997), assumed that the He I absorption arises on the irradiated hemisphere of the companion.

The Doppler map of $\mathrm{N}$ III $\lambda 4640$ yields a bright spot at $V_{y}=300 \pm 8 \mathrm{~km} \mathrm{~s}^{-1}$. Since this spot is produced on the inner hemisphere of the companion star through irradiation, it gives a lower limit to $K_{2}$, the velocity semiamplitude of the companion's center of mass. The lower limit to $K_{2}$ can then be combined with the very accurate determination of the inclination $(i=82.5 \pm 1.5)$ and the radial velocity curve of the pulsar to derive robust lower limits to the masses of the neutron star and companion of $\quad M_{1} \geq 1.14 \pm 0.06 M_{\odot} \quad$ and $\quad M_{2} \geq 0.36 \pm 0.02 M_{\odot}$, respectively.

An upper limit to $K_{2}$ can be obtained by using the $K$-correction equation:

$$
K_{2}=K_{\mathrm{em}} /\left[1-f q^{1 / 3}(1+q)^{2 / 3}\right]
$$

(Wade \& Horne 1988), where $K_{\mathrm{em}}$ is the velocity semiamplitude of the irradiation-induced emission, $q=M_{2} / M_{1}$, and $f \leq 1$ is the displacement of the reprocessing site from the center of mass of the star in units of the companion's star radius $R_{2}$. Furthermore, we assume that the $\mathrm{N}$ III line (with $K_{\mathrm{em}}=300 \mathrm{~km} \mathrm{~s}^{-1}$ ) is produced at the L1 point, i.e., $f=1$. The equation then yields $K_{2} \leq 437 \mathrm{~km} \mathrm{~s}^{-1}$, where we have used $q=K_{1} / K_{2}$ and $K_{1}=94.5 \mathrm{~km} \mathrm{~s}^{-1}$. This upper limit would imply $M_{1} \leq 3.1 M_{\odot}$, which coincides with the maximum neutron star mass possible with the stiffest equation of state of condensed matter. In addition, since $K_{2}$ must be between 300 and $437 \mathrm{~km} \mathrm{~s}^{-1}$ and we know $K_{1}$, this translates to firm limits to the mass ratio of $0.216<q<0.315$.

For the case of a canonical neutron star mass of $1.4 M_{\odot}$, we would expect $K_{2}=324 \mathrm{~km} \mathrm{~s}^{-1}$ and $q=0.29$. This would imply a $K$-correction for $\mathrm{N}$ III of $K_{\mathrm{em}} / K_{2}=0.93$, which is commensurate with the value seen for the cataclysmic variable HU Aqr, which has a similar mass ratio (Schwope, Mantel, \& Horne 1997). On the other hand, JvdK have shown that the neutron star in X1822-371 is undergoing an accretion spin-up phase, and therefore we might expect to find $M_{1}>1.4 M_{\odot}$. Tighter constraints on the system parameters may have important implications for our knowledge of the equation of state of nuclear matter (see, e.g., Cook, Shapiro, \& Teukolsky 1994).

The combination of light curves and velocity information from trailed spectra/Doppler maps has enabled us to locate the different regions responsible for the main spectral lines in the binary. The Balmer lines (from $\mathrm{H} \beta$ to the Balmer break) are embedded in deep absorptions whose phasing and large velocity shifts suggest that they are produced in vertically extended cool gas associated with the hot spot/ postshock region. Simulations of stream-disk interaction with inefficient cooling (Armitage \& Livio 1998) produce a "halo" of material above the disk and a bulge along the disk rim, which can obscure the bright inner disk. This behavior is mainly observed in SW Sex stars (see, e.g., Groot, Rutten, \& van Paradijs 2001; Martínez-Pais, Rodríguez-Gil, \& Casares 1999), where (possible) magnetic white dwarfs are fed by disk-overflowing material. SW Sex behavior has also been recognized in the neutron star transient XTE J2123-058 during outburst (Hynes et al. 2001). A key ingredient of this behavior seems to depend on large accretion rates, although an inclination selection effect may be needed to account for the lack of orbitally modulated absorptions in other persistent LMXBs (e.g., Sco X-1). The O VI $\lambda 3811$ emission arises from the postshock region between the gas stream and the disk, but it has maximum flux at phase 0.25 , rather than 0.8 . Therefore, it must be triggered by irradiation of the inner hot spot bulge region, i.e., further evidence for a vertical thickening of the disk at the splash point position.

After our work on Sco X-1 (SC) and GX 339-4 (Hynes et al. 2003), this paper further establishes the importance of the Bowen fluorescence as an excellent tracer of the companion's motion in active LMXBs and soft X-ray transients in outburst. This new technique opens a new window on the detection of the otherwise radiatively overwhelmed companion stars and thereby allows follow-up dynamical studies of X-ray binaries.

D. S. acknowledges the support of a PPARC Postdoctoral Fellowship and a Smithsonian Astrophysical Observatory Clay Fellowship. R. I. H. and P. A. C. acknowledge support from grant $\mathrm{F} / 00-180 / \mathrm{A}$ from the Leverhulme Trust. R. I. H. is currently funded from NASA through Hubble Fellowship grant HF-01150.01-A awarded by the Space Telescope Science Institute, which is operated by the Association of Universities for Research in Astronomy (AURA), Inc., for NASA, under contract NAS 526555. MOLLY and DOPPLER software developed by T. R. Marsh is gratefully acknowledged. This work is partly based on data collected at the European Southern Observatory, La Silla, Chile. 
Armitage, P. J., \& Livio, M. 1998, ApJ, 493, 898

Charles, P., Thorstensen, J. R., \& Barr, P. 1980, ApJ, 241, 1148

Cook, G. B., Shapiro, S. L., \& Teukolsky, S. A. 1994, ApJ, 424, 823

Groot, P. J., Rutten, R. G. M., \& van Paradijs, J. 2001, A\&A, 368, 183

Harlaftis, E. T., Charles, P. A., \& Horne, K. 1997, MNRAS, 285, 673

Heinz, S., \& Nowak, M. A. 2001, MNRAS, 320, 249

Hellier, C., \& Mason, K. O. 1989, MNRAS, 239, 715

Horne, K. 1986, PASP, 98, 609

Hynes, R. I., Charles, P. A., Haswell, C. A., Casares, J., Zurita, C., \& Serra-Ricart, M. 2001, MNRAS, 324, 180

Hynes, R. I., Steeghs, D., Casares, J., Charles, P. A., \& O’Brien, K. 2003, ApJ, 583, L95

Jonker, P. G., \& van der Klis, M. 2001, ApJ, 553, L43 (JvdK)

Jonker, P. G., van der Klis, M., \& Groot, P. J. 2003, MNRAS, 339, 663

Marsh, T. R. 2001, in Astrotomography, Indirect Imaging Methods in Observational Astronomy, ed. H. M. J. Boffin, D. Steeghs, \& J. Cuypers (LNP 573; Berlin: Springer), 1

\section{REFERENCES}

Marsh, T. R., Horne, K., Schlegel, E. M., Honeycutt, R. K., \& Kaitchuck, R. H. 1990, ApJ, 364, 637

Martínez-Pais, I. G., Rodríguez-Gil, P., \& Casares, J. 1999, MNRAS, 305, 661

Mason, K. O., \& Córdova, F. A. 1982, ApJ, 262, 253

Mason, K. O., Murdin, P. G., Tuohy, I. R., Seitzer, P., \& BranduardiRaymont, G. 1982, MNRAS, 200, 793

McClintock, J. E., Canizares, C. R., \& Tarter, C. B. 1975, ApJ, 198, 641

Parmar, A. N., Oosterbroek, T., Del Sordo, S., Segreto, A., Santangelo, A., Dal Fiume, D., \& Orlandini, M. 2000, A\&A, 356, 175

Schneider, D. P., \& Young, P. 1980, ApJ, 238, 946

Schwope, A. D., Mantel, K.-H., \& Horne, K. 1997, A\&A, 319, 894

Steeghs, D., \& Casares, J. 2002, ApJ, 568, 273 (SC)

van Paradijs, J., \& McClintock, J. E. 1995, in X-Ray Binaries, ed. W. H. G. Lewin, J. van Paradijs, \& E. P. J. van den Heuvel (Cambridge: Cambridge Univ. Press), 58

Wade, R. A., \& Horne, K. 1988, ApJ, 324, 411

White, N. A., \& Holt, S. S. 1982, ApJ, 257, 318 